\title{
Preliminary Findings of Radon Potential Indexes in Five Canadian Cities
}

\author{
J. Chen (Corresponding author), D. Moir, K. MacLellan, E. Leigh, D. Nunez \& S. Murphy \\ Radiation Protection Bureau, Health Canada, 2720 Riverside Drive \\ Ottawa, Ontario K1A 0K9, Canada \\ E-mail: jing.chen@hc-sc.gc.ca \\ K. Ford \\ Geological Survey of Canada, 601 Booth Street \\ Ottawa. Ontario K1A 0E8, Canada
}

Received: July 25, 2011

Accepted: August 12, $2011 \quad$ Published: March 1, 2012

doi:10.5539/enrr.v2n1p2

URL: http://dx.doi.org/10.5539/enrr.v2n1p2

\begin{abstract}
Radon has been identified as the second leading cause of lung cancer after tobacco smoking. Since radon in soil is believed to be the main source of radon in Canadian homes, a radon potential index determined from soil radon concentration and soil permeability can be used to describe the indoor radon potential resulting from radon in soil gas. The index increases with increasing radon concentration in soil gas and soil permeability. This study reports detailed measurements of soil gas radon concentrations and soil permeability in a total of 254 sites in five cities, Montreal, Gatineau, Ottawa, Kingston and Toronto. Average radon potential indexes were determined for each individual site of five measurement locations. The results provided additional data for the mapping of radon potentials in Canada.
\end{abstract}

Keywords: Radon, Soil gas, Soil permeability

\section{Introduction}

Radon is a naturally occurring radioactive gas generated by the decay of uranium-bearing minerals in rocks and soils. A certain fraction of the radon escapes from the ground into the air. In the open air, radon is diluted to low concentrations and is not a health concern. In indoor environments, radon originating as soil gas is the main source of natural radiation exposure to the population. Radon has been identified as the second leading cause of lung cancer after tobacco smoking (WHO, 2005).

Based on new scientific information and a broad public consultation, the Canadian radon guideline was lowered from 800 to $200 \mathrm{Bqm}^{-3}$ in June 2007 (Health Canada, 2007). To support the implementation of the revised guideline, a National Radon Program (NRP) was developed. A significant component of the NRP is the development of a radon database that will also include a number of targeted mapping activities. In Canada, radon maps are intended to be used mainly by governments to prioritize radon outreach and education efforts, to encourage testing and remediation where necessary, and to assist in community planning and future development. Canadian radon maps will be based on a variety of data, such as ground uranium concentration from airborne gamma ray spectrometric surveys, radon in soil gas, soil permeability, soil geochemistry, surficial geology, bedrock geology, and indoor radon data (Chen, 2009). To assist radon mapping, measurements of soil gas radon concentration and soil permeability have been conducted in cities since 2007. Determination of soil radon potential indexes in five cities (Montreal, Gatineau, Ottawa, Kingston \& Toronto) are reported here.

\section{Methods}

All surveys followed well established protocol of the National Soil Radon Project, a Canadian add-on project to the North American Soil Geochemical Landscapes Project (GSC, 2008). Surveys were conducted on a dry day 
with a clear sky at least the previous evening. Soil gas radon and soil permeability measurements were conducted in community parks within residential areas. The soil survey sites were areas of about $10 \times 10 \mathrm{~m}^{2}$ in low-traffic areas of community parks and away from roads. For each site, five soil gas radon measurements and at least two in-situ soil permeability measurements were performed with four probes at each corner and one in the centre of the $10 \times 10 \mathrm{~m}^{2}$ survey area.

Soil gas radon was determined by measuring the concentration of radon in soil gas samples extracted from $80 \mathrm{~cm}$ depth below ground surface using the RM-2 system manufactured by Radon v.o.s. in Czech Republic (http://www.radon.eu/rm2.html). Soil gas samples were collected using small-diameter hollow steel probes with a free, sharpened lower end (a lost tip) combined with a syringe. Soil gas samples of $150 \mathrm{ml}$ in a syringe were introduced into ionizing chambers for measurement of radon concentrations. An ionization chamber was only used when its background reading was below $0.7 \mathrm{kBq} \mathrm{m}^{-3}$, as instructed by the manufacture. Soil radon concentrations measured below $1 \mathrm{kBq} \mathrm{m}^{-3}$ were excluded. Any potential leakage during the soil gas sampling could result in a lower radon concentration. Therefore, the lowest measured value of soil gas radon was also excluded in the calculation of the average radon concentration for a site.

Soil gas permeability was measured $80 \mathrm{~cm}$ below ground surface with the use of RADON-JOK also manufactured by Radon in C.o.s. Republic (http://www.radon-vos.cz/?lang=en\&lmenu=en_measuring\&page=en_measuring_jok). RADON-JOK is based on air withdrawal by means of negative pressure. The soil gas is pumped under constant pressure through a probe (the same probe as used for soil radon collecting) with a constant surface, an active area created in the head of the probe at $80 \mathrm{~cm}$ below the ground. The soil gas permeability was calculated based on Darcy's equation (Koorevaar et al., 1983). For sites having very low permeability, the in-situ soil gas permeability measurement could potentially take hours to complete. For logistical reasons, in those cases, a default value of 2 $\cdot 10^{-14} \mathrm{~m}^{2}$ is assumed. In the city of Ottawa, in-situ permeability measurements were conducted in all five probes per site while permeability measurements were performed at two out of five probes at each site in other cities. For those sites where only two in-situ permeability measurements were performed, the semi-quantitative estimation of permeability was applied with consideration of how hard to collect soil gas with a syringe at other three probes. For each probe where a soil gas sample was collected with a syringe, a subjective description of how hard to collect soil gas with a syringe was recorded as easy, medium or hard. This provides a semi-quantitative estimation of permeability, especially for those values between in-situ measured permeability and the default value of $2 \cdot 10^{-14} \mathrm{~m}^{2}$.

Soil gas radon concentration and soil permeability are the two most important factors that affect radon flux from soil to air (Neznal et al., 2006). Since radon in soil is believed to be the main source of radon in Canadian homes, a radon potential index determined from soil radon concentration and soil permeability can be used to describe the indoor radon potential resulting from radon in soil gas. The index was also called soil radon potential (SRP) index in previous publications (Chen et al., 2008a, 2008b), that is defined as:

$$
S R P=\frac{C-C_{0}}{-\log (P)+\log \left(P_{0}\right)}
$$

where $\mathrm{C}$ is the radon concentration in soil gas in units of $\mathrm{kBq} \mathrm{m}^{-3}$, and $\mathrm{P}$ is the soil permeability in units of $\mathrm{m}^{2}$. $\mathrm{C}_{0}$ and $\mathrm{P}_{0}$ are set to $1 \mathrm{kBq} \mathrm{m}{ }^{-3}$ and $1 \cdot 10^{-10} \mathrm{~m}^{2}$, respectively. In this study, SRP indexes were calculated for each site surveyed.

\section{Results and Discussion}

For the sites where in-situ permeability measurements were performed in all five probes, an average permeability of the five measurements was assigned to that site. For the sites where only two permeability measurements were performed at each site of five probes for soil radon measurements, an average permeability of the two measurements was assigned to that site where at least one in-situ permeability measurements were successfully performed, i.e. above the default value for very low permeability $\left(2 \cdot 10^{-14} \mathrm{~m}^{2}\right)$. Some sites surveyed had very low permeability at both probes for in-situ measurement. For those sites, the semi-quantitative estimation of permeability is applied with consideration of how hard to collect soil gas with a syringe at other three probes, as given in a previous publication (Chen et al., 2011).

A total of 254 sites were surveyed with 76, 36, 42, 26 and 74 sites in Montreal, Gatineau, Ottawa, Kingston and Toronto, respectively. Soil radon concentrations varied significantly from site to site and also from probe to probe for most sites surveyed. Therefore, an average soil radon concentration was calculated for each site. With the average soil radon concentration and measured and/or assigned permeability, a soil radon potential (SRP) index was calculated to characterize each site. Summary results are given in Table 1. Since radon in soil is 
believed to be the main source of radon in Canadian homes, the indoor radon potential could have a strong association with the soil radon potential as given in Table 1 .

Indoor radon potential for a community could be represented by the percentage of homes above the Canadian guideline value of $200 \mathrm{Bqm}^{-3}$ in that community. It characterises the average radon level in a community, and can not be used to predict radon concentration in any individual homes. From Table 1, one can see that, within certain variations, percentages of homes above $200 \mathrm{Bqm}^{-3}$ could be comparable in the five cities surveyed. This prediction needs to be verified by direct indoor radon measurements when detailed results of the cross Canada radon survey (Health Canada, 2011) become available.

This paper is aimed to provide additional layer of data to the development of Canadian radon potential maps. For this purpose, detailed results for individual sites are given in Tables 2 to 6 for Montreal, Gatineau, Ottawa, Kingston and Toronto, respectively. It should be mentioned that soil radon potential is only one layer of data required in a multi-tier approach of indoor radon potential mapping. Canadian radon maps will be based on a variety of data. Results presented here can be used to fill data gaps in the map, especially in cities where airborne gamma ray spectrometric surveys were restricted.

\section{References}

Chen, J., Ly, J., Bergman, L., Wierdsma, J., \& Klassen, R. (2008a). Variation of soil radon concentrations in southern Ontario. Radiat. Prot. Dosim., 131, 385-389. http://dx.doi.org/10.1093/rpd/ncn192

Chen, J., Falcomer, R., Ly, J., Wierdsma, J., \& Bergman, L. (2008b). Long-term monitoring of soil gas radon and permeability at two reference sites. Radiat. Prot. Dosim., 131, 503-508. http://dx.doi.org/10.1093/rpd/ncn274

Chen, J. (2009). A preliminary Design of Radon Potential Map for Canada - a multi-tier approach Environ. Earth Sci., 59, 775-782. http://dx.doi.org/10.1007/s12665-009-0073-x

Chen, J., Moir, D. K., MacLellan, K., Leigh, E., Nunez, D., Murphy, S., \& Ford, K. (2011). Soil radon measurements in Canadian cities. Radiat. Prot. Dosim. In press.

Geological Survey of Canada. (2008). North American Soil Geochemical Landscapes Project. [Online] Available: http://sst.rncan.gc.ca/eh-esh/trinat/progress_e.php.

Health Canada. (2007). Government Canada Radon Guideline. [Online] Available: http://www.hc-sc.gc.ca/ewh-semt/radiation/radon/guidelines_lignes_directrice-eng.php.

Health Canada. (2011). Cross Canada Survey of Radon Concentrations in Homes - Year 1 Highlights. [Online] Available: http://www.hc-sc.gc.ca/ewh-semt/radiation/radon/survey-sondage-eng.php.

Koorevaar, P., Menelik, G., \& Dirksen, C. (1983). Elements of soil physics. Developments in soil science. Vol. 13. (Amsterdam/Oxford/New York/Tokyo: Elsevier).

Neznal, M., Neznal, M., Matolin, M., Barnet, I., \& Miksova, J. (2006). The new method for assessing the radon risk of building sites. Project report (State Office for Nuclear Safety).

The World Health Organization. (2005). Fact Sheet No.291: Radon and cancer, June 2005. [Online] Available: http://www.who.int/mediacentre/factsheets/fs291/en/index.html. (28 April 2008)

Table 1. Summary of results: averages and standard deviations including measurement ranges in brackets for soil radon surveys in Ottawa (2007) and in Montreal, Gatineau, Kingston and Toronto (2010)

\begin{tabular}{ccccc}
\hline city & sites & $\mathrm{C}(\mathrm{kBq} / \mathrm{m} 3)$ & $\mathrm{P}(\mathrm{m} 2)$ & SRP \\
\hline Montreal & 76 & $28 \pm 21(1,157)$ & $5.3 \cdot 10^{-12} \pm 5.5 \cdot 10^{-12}\left(2.0 \cdot 10^{-14}, 2.2 \cdot 10^{-11}\right)$ & $20 \pm 16(0,64)$ \\
Gatineau & 36 & $17 \pm 11(1,47)$ & $5.7 \cdot 10^{-12} \pm 6.6 \cdot 10^{-12}\left(4.1 \cdot 10^{-14}, 2.0 \cdot 10^{-11}\right)$ & $12 \pm 11(0,61)$ \\
Ottawa & 42 & $22 \pm 15(2,67)$ & $3.9 \cdot 10^{-12} \pm 6.0 \cdot 10^{-12}\left(2.0 \cdot 10^{-14}, 2.7 \cdot 10^{-11}\right)$ & $12 \pm 10(0,49)$ \\
Kingston & 26 & $11 \pm 10(1,42)$ & $4.0 \cdot 10^{-12} \pm 5.4 \cdot 10^{-12}\left(2.0 \cdot 10^{-14}, 1.8 \cdot 10^{-11}\right)$ & $8 \pm 9(0,35)$ \\
Toronto & 74 & $23 \pm 14(1,65)$ & $2.9 \cdot 10^{-12} \pm 3.7 \cdot 10^{-12}\left(2.0 \cdot 10^{-14}, 1.5 \cdot 10^{-11}\right)$ & $12 \pm 10(0,44)$ \\
\hline
\end{tabular}


Table 2. Soil radon survey results (average soil radon concentration in $\mathrm{kBq} / \mathrm{m}^{3}$, average permeability in $\mathrm{m}^{2}$, and SRP) in 76 community parks in Montreal

\begin{tabular}{|c|c|c|c|c|}
\hline Latitude & Longitude & Soil radon $\mathrm{kBq} / \mathrm{m}^{3}$ & Permeability $\mathrm{m}^{2}$ & SRP \\
\hline 45.30150 & $\begin{array}{l}-73.78040 \\
\end{array}$ & 24.7 & $9.8 \mathrm{E}-13$ & 11.8 \\
\hline 45.40587 & -73.95101 & 55.0 & $3.8 \mathrm{E}-12$ & 38.0 \\
\hline 45.41670 & -73.90944 & 8.9 & $2.0 \mathrm{E}-14$ & 2.1 \\
\hline 45.41976 & -73.64191 & 27.1 & $2.2 \mathrm{E}-12$ & 15.8 \\
\hline 45.42769 & -73.87747 & 26.9 & $1.3 \mathrm{E}-11$ & 29.5 \\
\hline 45.43219 & -73.62305 & 43.9 & $2.1 \mathrm{E}-11$ & 62.7 \\
\hline 45.43804 & -73.71603 & 18.7 & $8.9 \mathrm{E}-13$ & 8.6 \\
\hline 45.43880 & -73.67297 & 12.1 & $2.2 \mathrm{E}-11$ & 16.9 \\
\hline 45.43894 & -73.92964 & 1.3 & $2.0 \mathrm{E}-14$ & 0.1 \\
\hline 45.44001 & -73.74774 & 11.5 & $1.1 \mathrm{E}-12$ & 5.4 \\
\hline 45.44093 & -73.48617 & 28.8 & $4.9 \mathrm{E}-13$ & 12.0 \\
\hline 45.44133 & -73.90016 & 47.5 & $5.5 \mathrm{E}-12$ & 36.9 \\
\hline 45.44437 & -73.86008 & 21.5 & $4.6 \mathrm{E}-12$ & 15.4 \\
\hline 45.44860 & -73.75838 & 49.1 & $4.9 \mathrm{E}-12$ & 36.8 \\
\hline 45.44971 & -73.45987 & 60.6 & $9.7 \mathrm{E}-12$ & 58.8 \\
\hline 45.45118 & -73.82253 & 5.8 & $2.0 \mathrm{E}-14$ & 1.3 \\
\hline 45.45456 & -73.60612 & 24.2 & $2.0 \mathrm{E}-14$ & 6.3 \\
\hline 45.46059 & -73.89482 & 59.8 & $2.3 \mathrm{E}-12$ & 35.7 \\
\hline 45.46183 & -73.64533 & 14.9 & $3.4 \mathrm{E}-13$ & 5.6 \\
\hline 45.46385 & -73.85503 & 23.1 & $3.4 \mathrm{E}-12$ & 15.0 \\
\hline 45.47278 & -73.61444 & 49.8 & $4.7 \mathrm{E}-12$ & 36.7 \\
\hline 45.47398 & -73.67171 & 13.4 & $2.0 \mathrm{E}-14$ & 3.3 \\
\hline 45.48002 & -73.58657 & 21.9 & $1.5 \mathrm{E}-11$ & 25.7 \\
\hline 45.48237 & -73.64624 & 38.8 & $6.1 \mathrm{E}-13$ & 17.1 \\
\hline 45.48319 & -73.47215 & 16.5 & $2.7 \mathrm{E}-12$ & 9.9 \\
\hline 45.48679 & -73.45123 & 2.2 & $2.0 \mathrm{E}-14$ & 0.3 \\
\hline 45.49319 & -73.79730 & 17.4 & $1.0 \mathrm{E}-11$ & 16.3 \\
\hline 45.49770 & -73.68977 & 29.2 & 7.2E-12 & 24.7 \\
\hline 45.50104 & -73.39747 & 157.3 & $3.6 \mathrm{E}-13$ & 63.8 \\
\hline 45.50388 & -73.50397 & 21.0 & $2.3 \mathrm{E}-13$ & 7.6 \\
\hline 45.50505 & -73.63054 & 46.1 & $1.4 \mathrm{E}-11$ & 53.1 \\
\hline 45.50956 & -73.70312 & 31.2 & $3.4 \mathrm{E}-12$ & 20.6 \\
\hline 45.51095 & -73.83920 & 34.1 & $1.2 \mathrm{E}-11$ & 35.2 \\
\hline 45.51550 & -73.72444 & 43.6 & $1.2 \mathrm{E}-11$ & 45.5 \\
\hline 45.51637 & -73.58637 & 48.1 & $6.0 \mathrm{E}-12$ & 38.5 \\
\hline 45.52441 & -73.78796 & 7.5 & $7.0 \mathrm{E}-13$ & 3.0 \\
\hline 45.53009 & -73.77078 & 17.8 & $1.1 \mathrm{E}-11$ & 17.8 \\
\hline 45.53058 & -73.92921 & 18.1 & $4.8 \mathrm{E}-12$ & 12.9 \\
\hline 45.53273 & -73.44504 & 32.0 & $1.0 \mathrm{E}-11$ & 31.1 \\
\hline 45.53354 & -73.73245 & 15.5 & $2.2 \mathrm{E}-12$ & 8.7 \\
\hline 45.53640 & -73.68385 & 9.2 & $9.5 \mathrm{E}-12$ & 8.0 \\
\hline 45.53772 & -73.59417 & 18.0 & $2.2 \mathrm{E}-12$ & 10.3 \\
\hline 45.54038 & -73.50013 & 47.4 & $3.1 \mathrm{E}-13$ & 18.5 \\
\hline 45.54187 & -73.89613 & 14.2 & $6.8 \mathrm{E}-12$ & 11.3 \\
\hline 45.54707 & -73.61889 & 69.4 & $2.2 \mathrm{E}-12$ & 41.5 \\
\hline 45.54844 & -73.65089 & 17.6 & $1.2 \mathrm{E}-11$ & 18.0 \\
\hline 45.54925 & -73.54958 & 6.3 & $4.8 \mathrm{E}-12$ & 4.0 \\
\hline 45.55303 & -73.76755 & 22.8 & $1.3 \mathrm{E}-13$ & 7.6 \\
\hline 45.55455 & -73.70626 & 12.6 & $4.4 \mathrm{E}-13$ & 4.9 \\
\hline 45.55975 & -73.47765 & 24.1 & $5.8 \mathrm{E}-12$ & 18.6 \\
\hline 45.56041 & -73.89622 & 23.6 & 8.3E-12 & 20.9 \\
\hline 45.56310 & -73.55691 & 14.4 & $3.4 \mathrm{E}-12$ & 9.1 \\
\hline 45.57003 & -73.61687 & 20.5 & $1.0 \mathrm{E}-11$ & 19.5 \\
\hline 45.57098 & -73.72966 & 22.2 & $4.1 \mathrm{E}-13$ & 8.9 \\
\hline 45.57538 & -73.64436 & 15.1 & $3.1 \mathrm{E}-12$ & 9.4 \\
\hline
\end{tabular}




\begin{tabular}{ccccc}
45.57819 & -73.89899 & 43.4 & $5.3 \mathrm{E}-14$ & 12.9 \\
45.57867 & -73.67905 & 51.6 & $9.0 \mathrm{E}-12$ & 48.3 \\
45.58144 & -73.55377 & 9.7 & $4.3 \mathrm{E}-13$ & 3.7 \\
45.58376 & -73.60902 & 3.1 & $3.4 \mathrm{E}-12$ & 1.4 \\
45.58416 & -73.92610 & 23.5 & $2.0 \mathrm{E}-14$ & 6.1 \\
45.58490 & -73.86031 & 47.6 & $6.1 \mathrm{E}-13$ & 21.0 \\
45.59213 & -73.59074 & 39.9 & $1.3 \mathrm{E}-12$ & 20.5 \\
45.59382 & -73.44754 & 24.4 & $4.1 \mathrm{E}-12$ & 16.8 \\
45.59425 & -73.52003 & 34.2 & $1.8 \mathrm{E}-13$ & 12.1 \\
45.60024 & -73.63714 & 29.5 & $5.0 \mathrm{E}-12$ & 21.9 \\
45.60196 & -73.65794 & 41.1 & $1.4 \mathrm{E}-11$ & 46.4 \\
45.60431 & -73.56923 & 17.4 & $3.6 \mathrm{E}-12$ & 11.3 \\
45.60814 & -73.53729 & 41.1 & $1.3 \mathrm{E}-11$ & 45.2 \\
45.61039 & -73.59530 & 20.4 & $9.6 \mathrm{E}-12$ & 19.1 \\
45.61565 & -73.62405 & 25.6 & $1.1 \mathrm{E}-11$ & 25.6 \\
45.62732 & -73.59251 & 19.1 & $9.4 \mathrm{E}-12$ & 17.6 \\
45.63969 & -73.49861 & 19.9 & $3.2 \mathrm{E}-12$ & 12.6 \\
45.64398 & -73.58823 & 16.6 & $1.0 \mathrm{E}-11$ & 15.9 \\
45.65448 & -74.09345 & 16.7 & $1.7 \mathrm{E}-13$ & 5.7 \\
45.65529 & -73.50076 & 26.0 & $2.3 \mathrm{E}-12$ & 15.2 \\
45.66244 & -73.55072 & 27.6 & $2.2 \mathrm{E}-11$ & 40.6 \\
\hline
\end{tabular}

Table 3. Soil radon survey results (average soil radon concentration in $\mathrm{kBq} / \mathrm{m}^{3}$, average permeability in $\mathrm{m}^{2}$, and SRP) in 36 community parks in Gatineau

\begin{tabular}{ccccc}
\hline Latitude & Longitude & Soil radon $\mathrm{kBq} / \mathrm{m}^{3}$ & Permeability $\mathrm{m}^{2}$ & SRP \\
\hline 45.38678 & -75.84184 & 14.5 & $2.0 \mathrm{E}-11$ & 19.1 \\
45.39283 & -75.82639 & 29.7 & $1.5 \mathrm{E}-13$ & 10.1 \\
45.39747 & -75.80582 & 6.2 & $1.2 \mathrm{E}-11$ & 5.7 \\
45.40888 & -75.79626 & 24.9 & $3.9 \mathrm{E}-13$ & 9.9 \\
45.41597 & -75.84550 & 18.8 & $1.6 \mathrm{E}-11$ & 22.0 \\
45.41943 & -75.75222 & 15.5 & $7.8 \mathrm{E}-12$ & 13.1 \\
45.42370 & -75.43270 & 1.0 & $4.1 \mathrm{E}-14$ & 0.0 \\
45.42482 & -75.75936 & 30.4 & $4.2 \mathrm{E}-12$ & 21.4 \\
45.42628 & -75.73347 & 12.8 & $1.8 \mathrm{E}-11$ & 15.8 \\
45.43012 & -75.71562 & 13.2 & $2.9 \mathrm{E}-12$ & 7.9 \\
45.43458 & -75.70768 & 23.6 & $3.4 \mathrm{E}-12$ & 15.4 \\
45.43724 & -75.79552 & 1.0 & $6.1 \mathrm{E}-13$ & 0.0 \\
45.43803 & -75.72375 & 7.5 & $3.4 \mathrm{E}-12$ & 4.4 \\
45.44196 & -75.78279 & 13.9 & $3.4 \mathrm{E}-13$ & 5.2 \\
45.44271 & -75.77666 & 11.4 & $1.6 \mathrm{E}-12$ & 5.8 \\
45.45326 & -75.74669 & 31.8 & $4.1 \mathrm{E}-12$ & 22.3 \\
45.45481 & -75.75191 & 34.1 & $2.6 \mathrm{E}-13$ & 12.8 \\
45.45612 & -75.42739 & 7.6 & $9.9 \mathrm{E}-12$ & 6.5 \\
45.46356 & -75.75406 & 16.7 & $4.5 \mathrm{E}-13$ & 6.7 \\
45.46471 & -75.07679 & 18.2 & $2.7 \mathrm{E}-12$ & 11.0 \\
45.47722 & -75.68531 & 28.2 & $3.1 \mathrm{E}-12$ & 18.1 \\
45.47852 & -75.63909 & 14.4 & $1.4 \mathrm{E}-11$ & 15.7 \\
45.48140 & -75.48140 & 36.6 & $1.1 \mathrm{E}-13$ & 12.0 \\
45.48678 & -75.48678 & 19.3 & $1.1 \mathrm{E}-13$ & 6.2 \\
45.49138 & -75.69865 & 2.7 & $1.1 \mathrm{E}-13$ & 0.6 \\
45.49157 & -75.59798 & 9.0 & $1.1 \mathrm{E}-13$ & 2.7 \\
45.50209 & -75.68008 & 3.6 & $6.1 \mathrm{E}-13$ & 1.2 \\
45.50438 & -75.65306 & 4.9 & $3.4 \mathrm{E}-12$ & 2.6 \\
45.50454 & -75.60797 & 21.6 & $3.2 \mathrm{E}-12$ & 13.8 \\
45.50556 & -75.59229 & 16.4 & $7.0 \mathrm{E}-12$ & 13.3 \\
45.50748 & -75.57867 & 10.8 & $5.1 \mathrm{E}-12$ & 7.5 \\
45.52714 & -75.64064 & 1.0 & $3.4 \mathrm{E}-12$ & 0.0 \\
45.52861 & -75.91411 & 47.0 & $1.8 \mathrm{E}-11$ & 61.0 \\
& & & &
\end{tabular}




\begin{tabular}{ccccc}
45.53447 & -75.82533 & 22.5 & $1.9 \mathrm{E}-11$ & 29.9 \\
45.58932 & -75.90336 & 16.2 & $1.7 \mathrm{E}-12$ & 8.7 \\
45.86171 & -75.40004 & 13.5 & $2.0 \mathrm{E}-11$ & 17.7 \\
\hline
\end{tabular}

Table 4. Soil radon survey results (average soil radon concentration in $\mathrm{kBq} / \mathrm{m}^{3}$, average permeability in $\mathrm{m}^{2}$, and SRP) in 42 community parks in Ottawa

\begin{tabular}{ccccc}
\hline Latitude & Longitude & Soil radon $\mathrm{kBq} / \mathrm{m}^{3}$ & Permeability $\mathrm{m}^{2}$ & SRP \\
\hline 45.13938 & -75.60484 & 2.4 & $5.6 \mathrm{E}-12$ & 1.1 \\
45.19650 & -75.83875 & 32.6 & $9.6 \mathrm{E}-14$ & 10.5 \\
45.22243 & -75.68892 & 40.0 & $1.2 \mathrm{E}-12$ & 20.2 \\
45.23742 & -75.47831 & 29.8 & $1.9 \mathrm{E}-12$ & 16.7 \\
45.24482 & -75.59020 & 66.8 & $4.4 \mathrm{E}-12$ & 48.6 \\
45.24509 & -75.70149 & 29.4 & $2.9 \mathrm{E}-13$ & 11.2 \\
45.24937 & -75.59959 & 4.3 & $2.0 \mathrm{E}-14$ & 0.9 \\
45.26222 & -75.94209 & 6.4 & $3.5 \mathrm{E}-12$ & 3.7 \\
45.26739 & -75.91285 & 12.8 & $2.3 \mathrm{E}-12$ & 7.2 \\
45.26797 & -75.82615 & 38.9 & $3.4 \mathrm{E}-12$ & 25.8 \\
45.27486 & -75.69083 & 22.3 & $1.3 \mathrm{E}-13$ & 7.4 \\
45.27795 & -75.85137 & 14.7 & $2.3 \mathrm{E}-14$ & 3.8 \\
45.28189 & -75.76294 & 31.8 & $1.8 \mathrm{E}-13$ & 11.2 \\
45.28757 & -75.71651 & 18.8 & $2.5 \mathrm{E}-13$ & 6.8 \\
45.29297 & -75.87672 & 22.6 & $9.5 \mathrm{E}-14$ & 7.2 \\
45.30376 & -75.90224 & 24.1 & $1.3 \mathrm{E}-11$ & 25.6 \\
45.31915 & -75.82758 & 28.8 & $3.0 \mathrm{E}-12$ & 18.3 \\
45.32766 & -75.89394 & 25.5 & $2.9 \mathrm{E}-14$ & 6.9 \\
45.32770 & -75.78418 & 25.6 & $2.1 \mathrm{E}-13$ & 9.2 \\
45.33664 & -75.60621 & 27.3 & $5.9 \mathrm{E}-12$ & 21.4 \\
45.34114 & -75.76836 & 22.9 & $1.6 \mathrm{E}-13$ & 7.8 \\
45.34118 & -75.63457 & 10.8 & $1.6 \mathrm{E}-11$ & 12.3 \\
45.34984 & -76.03506 & 17.2 & $1.8 \mathrm{E}-11$ & 21.4 \\
45.35117 & -75.92391 & 58.6 & $9.8 \mathrm{E}-14$ & 19.1 \\
45.35170 & -75.62634 & 16.0 & $8.3 \mathrm{E}-12$ & 13.9 \\
45.35212 & -75.94211 & 64.8 & $1.1 \mathrm{E}-12$ & 32.4 \\
45.35295 & -75.72057 & 11.9 & $2.4 \mathrm{E}-13$ & 4.2 \\
45.35709 & -75.78975 & 19.6 & $2.3 \mathrm{E}-12$ & 11.3 \\
45.36686 & -75.67605 & 10.3 & $2.0 \mathrm{E}-14$ & 2.5 \\
45.37131 & -75.72701 & 21.2 & $7.6 \mathrm{E}-12$ & 18.1 \\
45.38297 & -75.71412 & 12.4 & $1.8 \mathrm{E}-12$ & 6.5 \\
45.39597 & -75.65003 & 29.7 & $1.8 \mathrm{E}-12$ & 16.5 \\
45.39830 & -75.69313 & 11.1 & $1.4 \mathrm{E}-11$ & 12.0 \\
45.39857 & -75.75342 & 9.8 & $7.8 \mathrm{E}-12$ & 8.0 \\
45.40779 & -75.47556 & 33.1 & $8.9 \mathrm{E}-14$ & 8.8 \\
45.41925 & -75.42059 & 27.2 & $2.7 \mathrm{E}-11$ & 12.8 \\
45.43303 & -75.66850 & 6.8 & $2.0 \mathrm{E}-14$ & 10.3 \\
45.43745 & -75.52745 & 6.8 & $1.2 \mathrm{E}-11$ & 23.6 \\
45.44819 & -75.68486 & 22.5 & $2.3 \mathrm{E}-13$ & 4.0 \\
45.46217 & -75.46953 & 11.6 & $2.0 \mathrm{E}-14$ & 0.8 \\
45.46473 & -75.48168 & 4.0 & & 0.5 \\
45.47072 & -75.47569 & 2.8 & &
\end{tabular}


Table 5. Soil radon survey results (average soil radon concentration in $\mathrm{kBq} / \mathrm{m}^{3}$, average permeability in $\mathrm{m}^{2}$, and SRP) in 26 community parks in Kingston

\begin{tabular}{ccccc}
\hline Latitude & Longitude & Soil radon $\mathrm{kBq} / \mathrm{m}^{3}$ & Permeability $\mathrm{m}^{2}$ & SRP \\
\hline 44.21220 & -76.57608 & 5.2 & $3.4 \mathrm{E}-12$ & 2.8 \\
44.22547 & -76.48976 & 21.1 & $4.7 \mathrm{E}-12$ & 15.1 \\
44.22940 & -76.58295 & 19.8 & $3.4 \mathrm{E}-12$ & 12.8 \\
44.23313 & -76.49822 & 1.9 & $3.4 \mathrm{E}-12$ & 0.6 \\
44.23347 & -76.62688 & 8.7 & $2.0 \mathrm{E}-14$ & 2.1 \\
44.23378 & -76.51591 & 1.6 & $2.0 \mathrm{E}-14$ & 0.1 \\
44.23600 & -76.59685 & 24.8 & $5.8 \mathrm{E}-12$ & 19.2 \\
44.24282 & -76.48576 & 7.2 & $1.4 \mathrm{E}-11$ & 7.2 \\
44.24408 & -76.58569 & 31.6 & $1.4 \mathrm{E}-11$ & 35.2 \\
44.24673 & -76.54391 & 1.4 & $1.1 \mathrm{E}-13$ & 0.1 \\
44.24808 & -76.60774 & 4.0 & $1.1 \mathrm{E}-13$ & 1.0 \\
44.25007 & -76.50255 & 20.4 & $2.0 \mathrm{E}-14$ & 5.2 \\
44.25039 & -76.48431 & 15.6 & $2.6 \mathrm{E}-13$ & 5.7 \\
44.25314 & -76.56139 & 1.2 & $2.0 \mathrm{E}-14$ & 0.1 \\
44.25512 & -76.45796 & 3.4 & $1.1 \mathrm{E}-13$ & 0.8 \\
44.25569 & -76.38628 & 42.4 & $3.4 \mathrm{E}-12$ & 28.2 \\
44.25691 & -76.52547 & 8.3 & $1.3 \mathrm{E}-11$ & 8.3 \\
44.26266 & -76.57763 & 15.9 & $1.8 \mathrm{E}-11$ & 19.8 \\
44.26642 & -76.48907 & 11.7 & $6.3 \mathrm{E}-13$ & 4.8 \\
44.26657 & -76.44809 & 7.2 & $1.1 \mathrm{E}-13$ & 2.1 \\
44.27066 & -76.61889 & 17.2 & $6.4 \mathrm{E}-12$ & 13.6 \\
44.27283 & -76.45376 & 7.0 & $1.1 \mathrm{E}-11$ & 6.1 \\
44.30674 & -76.46206 & 8.9 & $1.2 \mathrm{E}-13$ & 2.7 \\
44.31048 & -76.52122 & 2.8 & $2.0 \mathrm{E}-14$ & 0.5 \\
44.31144 & -76.44620 & 2.4 & $3.4 \mathrm{E}-12$ & 1.0 \\
44.31862 & -76.50040 & 1.8 & $1.1 \mathrm{E}-13$ & 0.3 \\
\hline
\end{tabular}

Table 6 . Soil radon survey results (average soil radon concentration in $\mathrm{kBq} / \mathrm{m}^{3}$, average permeability in $\mathrm{m}^{2}$, and SRP) in 74 community parks in Toronto

\begin{tabular}{ccccc}
\hline Latitude & Longitude & Soil radon $\mathrm{kBq} / \mathrm{m}^{3}$ & Permeability $\mathrm{m}^{2}$ & SRP \\
\hline 43.34579 & -79.33396 & 18.0 & $1.8 \mathrm{E}-12$ & 9.7 \\
43.60918 & -79.52940 & 38.7 & $3.4 \mathrm{E}-12$ & 25.7 \\
43.61890 & -79.66148 & 21.5 & $1.9 \mathrm{E}-12$ & 11.8 \\
43.62470 & -79.50678 & 24.8 & $6.2 \mathrm{E}-13$ & 10.8 \\
43.63042 & -79.57863 & 65.4 & $3.4 \mathrm{E}-12$ & 43.9 \\
43.63816 & -79.51971 & 11.8 & $3.0 \mathrm{E}-12$ & 7.1 \\
43.64351 & -79.49246 & 16.4 & $1.1 \mathrm{E}-12$ & 7.9 \\
43.64540 & -79.47480 & 6.1 & $1.2 \mathrm{E}-11$ & 5.6 \\
43.64943 & -79.41703 & 23.8 & $2.3 \mathrm{E}-12$ & 13.9 \\
43.65176 & -79.54321 & 20.1 & $6.0 \mathrm{E}-13$ & 8.6 \\
43.65937 & -79.50150 & 14.7 & $1.5 \mathrm{E}-12$ & 7.4 \\
43.66014 & -79.58431 & 27.0 & $2.0 \mathrm{E}-14$ & 7.0 \\
43.66410 & -79.39142 & 9.0 & $6.0 \mathrm{E}-12$ & 6.5 \\
43.66565 & -79.43405 & 11.3 & $3.7 \mathrm{E}-12$ & 7.2 \\
43.66567 & -79.36214 & 6.0 & $3.7 \mathrm{E}-12$ & 3.5 \\
43.66836 & -79.47835 & 9.7 & $5.2 \mathrm{E}-12$ & 6.8 \\
43.66945 & -79.32852 & 1.0 & $2.0 \mathrm{E}-14$ & 0.0 \\
43.67371 & -79.45970 & 15.4 & $4.6 \mathrm{E}-13$ & 6.1 \\
43.67581 & -79.49953 & 12.5 & $2.5 \mathrm{E}-12$ & 7.2 \\
43.68326 & -79.41036 & 15.3 & $1.8 \mathrm{E}-12$ & 8.1 \\
43.68347 & -79.42651 & 40.2 & $1.8 \mathrm{E}-12$ & 22.4 \\
43.68577 & -79.37061 & 23.9 & $1.8 \mathrm{E}-13$ & 8.3 \\
43.68750 & -79.46001 & 38.8 & $7.2 \mathrm{E}-14$ & 12.0 \\
43.68917 & -79.56194 & 4.3 & $2.0 \mathrm{E}-14$ & 0.9
\end{tabular}




\begin{tabular}{|c|c|c|c|c|}
\hline 43.69247 & -79.26651 & 15.8 & 8.4E-12 & 13.8 \\
\hline 43.69752 & -79.40038 & 14.5 & $1.5 \mathrm{E}-11$ & 16.1 \\
\hline 43.69876 & -79.31641 & 11.8 & $5.1 \mathrm{E}-12$ & 8.3 \\
\hline 43.70271 & -79.33817 & 17.4 & $1.5 \mathrm{E}-13$ & 5.8 \\
\hline 43.70290 & -79.54681 & 26.3 & $4.0 \mathrm{E}-13$ & 10.5 \\
\hline 43.70382 & -79.27402 & 22.3 & $3.1 \mathrm{E}-12$ & 14.1 \\
\hline 43.70566 & -79.29594 & 11.5 & 7.7E-12 & 9.4 \\
\hline 43.70633 & -79.51646 & 22.9 & $1.0 \mathrm{E}-12$ & 11.0 \\
\hline 43.70899 & -79.45675 & 17.0 & $1.2 \mathrm{E}-12$ & 8.3 \\
\hline 43.71018 & -79.37551 & 11.1 & $3.4 \mathrm{E}-13$ & 4.1 \\
\hline 43.71045 & -79.62254 & 12.0 & $8.1 \mathrm{E}-14$ & 3.5 \\
\hline 43.71305 & -79.26787 & 9.4 & $1.9 \mathrm{E}-13$ & 3.1 \\
\hline 43.71466 & -79.41013 & 31.8 & $1.7 \mathrm{E}-12$ & 17.4 \\
\hline 43.71653 & -79.49280 & 1.9 & $2.0 \mathrm{E}-14$ & 0.2 \\
\hline 43.71664 & -79.55861 & 48.0 & $1.1 \mathrm{E}-12$ & 23.9 \\
\hline 43.71784 & -79.29843 & 24.8 & $4.9 \mathrm{E}-13$ & 10.3 \\
\hline 43.72232 & -79.53916 & 2.5 & $5.9 \mathrm{E}-13$ & 0.7 \\
\hline 43.72414 & -79.57750 & 1.3 & $3.4 \mathrm{E}-12$ & 0.2 \\
\hline 43.72699 & -79.63577 & 14.9 & 3.4E-12 & 9.5 \\
\hline 43.72956 & -79.39249 & 42.1 & 7.6E-13 & 19.4 \\
\hline 43.73623 & -79.50703 & 45.7 & 6.3E-12 & 37.3 \\
\hline 43.73721 & -79.55965 & 15.8 & $1.5 \mathrm{E}-12$ & 8.2 \\
\hline 43.73842 & -79.20522 & 23.7 & $6.5 \mathrm{E}-13$ & 10.4 \\
\hline 43.73863 & -79.59448 & 31.0 & $1.1 \mathrm{E}-12$ & 15.4 \\
\hline 43.74288 & -79.27210 & 32.5 & $3.4 \mathrm{E}-12$ & 21.5 \\
\hline 43.74616 & -79.43920 & 43.3 & $6.9 \mathrm{E}-12$ & 36.5 \\
\hline 43.74857 & -79.54277 & 37.3 & $2.8 \mathrm{E}-12$ & 23.4 \\
\hline 43.75008 & -79.33860 & 21.1 & $1.4 \mathrm{E}-11$ & 23.9 \\
\hline 43.75009 & -79.48457 & 12.0 & $1.1 \mathrm{E}-13$ & 3.7 \\
\hline 43.75051 & -79.31631 & 10.5 & $1.0 \mathrm{E}-12$ & 4.7 \\
\hline 43.75105 & -79.60550 & 17.0 & $2.0 \mathrm{E}-14$ & 4.3 \\
\hline 43.75294 & -79.39159 & 24.1 & $4.3 \mathrm{E}-13$ & 9.8 \\
\hline 43.75534 & -79.49978 & 30.7 & $7.2 \mathrm{E}-13$ & 13.9 \\
\hline 43.76334 & -79.57137 & 8.7 & 7.3E-12 & 6.8 \\
\hline 43.77104 & -79.51894 & 48.8 & $1.9 \mathrm{E}-13$ & 17.5 \\
\hline 43.77188 & -79.43933 & 46.1 & $6.8 \mathrm{E}-12$ & 38.7 \\
\hline 43.77693 & -79.15668 & 43.0 & $5.9 \mathrm{E}-12$ & 34.1 \\
\hline 43.77731 & -79.21012 & 56.8 & $1.1 \mathrm{E}-13$ & 18.8 \\
\hline 43.77766 & -79.30016 & 30.1 & $9.2 \mathrm{E}-12$ & 28.2 \\
\hline 43.78058 & -79.35970 & 18.8 & 1.3E-11 & 20.2 \\
\hline 43.78201 & -79.31683 & 6.6 & $2.0 \mathrm{E}-14$ & 1.5 \\
\hline 43.78511 & -79.44293 & 27.9 & $2.0 \mathrm{E}-13$ & 10.0 \\
\hline 43.78767 & -79.37325 & 52.7 & $1.2 \mathrm{E}-13$ & 17.6 \\
\hline 43.79105 & -79.79105 & 15.7 & $1.8 \mathrm{E}-12$ & 8.4 \\
\hline 43.79606 & -79.40191 & 35.6 & $3.9 \mathrm{E}-13$ & 14.4 \\
\hline 43.80942 & -79.17261 & 20.0 & $1.3 \mathrm{E}-11$ & 21.7 \\
\hline 43.81607 & -79.32127 & 25.2 & $2.0 \mathrm{E}-12$ & 14.3 \\
\hline 43.81748 & -79.31017 & 26.9 & $6.9 \mathrm{E}-13$ & 12.0 \\
\hline 43.82333 & -79.27926 & 17.4 & 1.3E-13 & 5.7 \\
\hline 43.83133 & -79.23157 & 7.5 & $2.0 \mathrm{E}-14$ & 1.7 \\
\hline
\end{tabular}

